\title{
Pleiomorphic viruses revealed by cryo tomography: the structure of coronaviruses
}

\author{
M. Bárcena $^{1}$, W. Baterlink ${ }^{2}$, G.T. Oostergetel ${ }^{3}$, A. Verklijk $^{4}$, P.J. M. Rottier ${ }^{2}$,
} A.J. Koster ${ }^{1}$ and B.J. Bosch ${ }^{2}$

1. EM Section, Molecular Cell Biology, Leiden University Medical Center, P.O.Box 9600, 2300 RC, Leiden, The Netherlands

2. Virology Division, Infectious Diseases and Immunology, Faculty of Veterinary, Utrecht University, 3584 CL Utrecht, The Netherlands

3. Biophysical Chemistry, Groningen Biomolecular Sciences and Biotechnology Institute, University of Groningen, Nijenborgh 4, 9497 AG, Groningen, The Netherlands

4. Molecular Cell Biology, Utrecht University, Padualaan 8, $3584 \mathrm{CH}$ Utrecht, The Netherlands

m.barcena@lumc.nl

Keywords: cryo-electron tomography, coronavirus, murine hepatitis virus.

Cryo-electron microscopy has provided in the last decades a wealth of threedimensional information on viral structures. However, most of this knowledge stems from single particle methods, which rely on averaging and are therefore restricted to viral homogenous structures such as icosahedral capsids [1]. This type of analysis excludes pleiomorphic viruses, that is, viruses that, having defined general architectures, arrange their components into different shapes or sizes. Cryo-electron tomography, which provides three-dimensional reconstructions of unique specimens, is now starting to shed light into the structure of this type of viruses [2].

Coronaviruses (CoVs) are a good example of the difficulties posed by pleiomorphic viruses. CoVs are enveloped viruses containing the largest reported RNA genome [3,4]. A lot of attention has been drawn to these viruses since the outbreak in 2002-2003 of SARS (Severe Acute Respiratory Syndrome), whose causative agent was found to be a member of this group. In spite of the interest in CoVs, our structural understanding of them is rather limited, with basic question such as their internal arrangement still unclear. Previous works have been based on 2D electron (cryo)microscopy of complete/disrupted virions and were hampered by their pleiomorphic nature.

To directly investigate the global 3D structural organization of intact coronaviruses, we have applied cryo-electron tomography on purified samples of murine hepatitis virus (MHV), the prototype for CoVs. Seven tilt series were collected in a $300 \mathrm{kV}$ FEI Polara microscope equipped with a post-column energy filter. Each tilt series covered an angular range of $\sim 130^{\circ}$ in $2^{\circ}$ increments. The nominal underfocus value used was $4 \mu \mathrm{m}$, which ensured no contrast inversions due to the contrast transfer function up to about 3 $\mathrm{nm}$. The pixel size was $0.58 \mathrm{~nm}$ at the specimen level. In the tomograms, a total of about 140 viruses were reconstructed and independently examined in 3D. 
The reconstructed MHVs show a distinct spherical shape and a relatively homogenous size ( $85 \mathrm{~nm}$ envelope diameter, $\mathrm{SD} \pm 6 \mathrm{~nm}$ ), although viruses from $65 \%$ to $200 \%$ the volume of the average MHV were measured. Coiled structures and tubular fragments are observed in the interior of the virus in agreement with a helical RNP nucleocapsid model. The reconstructions provide no evidence of a shelled core. Instead, the RNP seems to be extensively folded into itself in compact structure that tends to closely follow the envelope. The viral envelope was found to be significantly thicker than a lipid bilayer and focal pairs revealed the existence of an extra layer, most likely formed by the C-terminal domains of the major envelope protein. Focal contact points and thread-like densities connecting the envelope and the RNP were revealed in the tomograms. Our results suggest a model for the structure of coronaviruses based upon local structural motifs that might serve as the basic framework for the global architecture of the virion.

1. T.S. Baker, N.H. Olson, and S.D. Fuller, Microbiol. Mol. Biol. Rev. 63 (1999) 862.

2. K. Grunewald and M. Cyrklaff, Curr. Opin. Microbiol. 9 (2006), 437.

3. C. A. M. de Haan and P. Rottier, Adv. Vir. Res. 64 (2005), 165.

4. P. S. Masters, Adv. Vir. Res. 66 (2006), 193.

5. Acknowledgments. This work was partially supported by a Marie Curie Intra-European fellowship to M.B. (MEIF-CT-2004-501540) 